The University of San Francisco

USF Scholarship: a digital repository @ Gleeson Library | Geschke Center

Nursing and Health Professions Faculty Research and Publications

2010

\title{
Comprehensive Evidence-Based Program Teaching Self-Management of Auditory Hallucinations on Inpatient Psychiatric Units
}

Robin Buccheri

University of San Francisco, buccherir@usfca.edu

L Trygstad

MBuffum

KLyttle

G Dowling

Follow this and additional works at: http://repository.usfca.edu/nursing_fac

Part of the Nursing Commons, and the Psychiatric and Mental Health Commons

\section{Recommended Citation}

Buccheri, R., Trygstad, L., Buffum, M., Lyttle, K., \& Dowling, G. (2010). Comprehensive evidence-based program teaching selfmanagement of auditory hallucinations on inpatient psychiatric units. Issues in Mental Health Nursing, 31(3), 223-231.

This Article is brought to you for free and open access by the School of Nursing and Health Professions at USF Scholarship: a digital repository @ Gleeson Library | Geschke Center. It has been accepted for inclusion in Nursing and Health Professions Faculty Research and Publications by an authorized administrator of USF Scholarship: a digital repository@ Gleeson Library | Geschke Center. For more information, please contact repository@usfca.edu. 


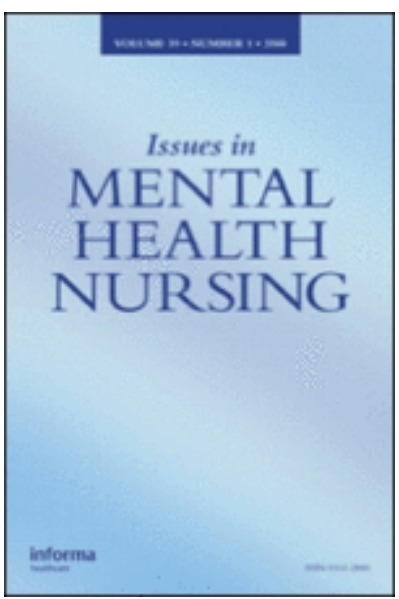

Comprehensive Evidence-based Program Teaching SelfManagement of Auditory Hallucinations on Inpatient Psychiatric Units

\begin{tabular}{|r|l|}
\hline Journal: & Issues in Mental Health Nursing \\
\hline Manuscript ID: & UMHN-2009-0071.R1 \\
\hline Manuscript Type: & Clinical Papers \\
\hline Keywords: & Auditory Hallucinations, Self-Management, Schizophrenia, Inpatient \\
\hline \multicolumn{2}{|c}{} \\
\hline
\end{tabular}

\section{今 scholaroNE" \\ Manuscript Central}


Comprehensive Program Auditory Hallucinations

2

3

4

5

6

7

8

9

10

11

12

13

14

15

16

17

18

19

20

21

22

23

24

25

26

27

28

29

30

31

32

33

34

35

36

37

38

39

40

41

42

43

44

45

46

47

48

49

50

51

52

53

54

55

56

57

58

59

60

\title{
Comprehensive Evidence-based Program Teaching
}

\section{Self-Management of Auditory Hallucinations on Inpatient Psychiatric Units}

\begin{abstract}
Patients hearing command hallucinations to harm whose only self-management strategies are to obey them, can represent serious safety concerns on inpatient psychiatric units. A comprehensive evidence-based program teaching self-management of auditory hallucinations on inpatient psychiatric units is described that includes five components: suggestions for staff education; patient self-assessment tools, interview guide and safety protocol; course to teach strategies for managing distressing voices and commands to harm; suggestions to improve staff communication; and a plan to extend the program from inpatient care settings into the community by sharing materials with community case managers and/or caregivers when patients are discharged.
\end{abstract}


Comprehensive Program Auditory Hallucinations

\section{Comprehensive Evidence-based Program Teaching}

\section{Self-Management of Auditory Hallucinations on Inpatient Psychiatric Units}

Many patients with schizophrenia on inpatient psychiatric units suffer from distressing auditory hallucinations. These auditory hallucinations often give "bad advice" including commanding patients to harm themselves or others. These voices are especially dangerous if patients lack effective self-management skills and think that the only way they can manage them is by obeying them (Buccheri, Trygstad, \& Dowling, 2007).

The purpose of this paper is to describe a five component evidence-based program to assess and manage auditory hallucinations on inpatient units. This program consists of: 1) ideas for increased awareness and education of staff; 2) self-assessment tools for patients and an interview guide for staff; 3) an evidence-based nursing intervention (10-session course to teach strategies to self-manage auditory hallucinations); 4) recommendations for improving staff-patient communication; and 5) a plan for sharing this information with caregivers when patients are discharged. Specific recommendations that address how each component of this comprehensive program can be fully integrated into inpatient psychiatric care are provided.

\section{Management Program}

The program is organized into five components. A description of each component is provided.

\section{Staff Education}

The education begins by increasing staff awareness about the experience of hearing voices. Increasing awareness can done in a variety of ways: 1) providing a reading list of articles about the experience of hearing voices written by people who hear voices; 2) providing a simulated 
Comprehensive Program Auditory Hallucinations

hearing voices experience (e.g., virtual reality experiences such as getting on a bus while experiencing hallucinations) that is available from Janssen--Division of Ortho-McNeil-Janssen Pharmaceuticals, Inc. or listening to a CD that simulates hearing voices while the staff member is doing various things in the community such as asking for directions or to use a restroom (Hearing Voices Curriculum available from the National Endowment Center, n.d.). These tools can help staff become more aware of the difficulties and confusion patients experience when simultaneously hearing voices and trying to answer questions and/or follow simple directions.

Increasing awareness is followed by providing education about the evidence-based practice of assessing and managing auditory hallucinations. This is accomplished by providing a reading list for staff about research that has been done on managing auditory hallucinations, having a staff in-service to discuss these articles, and if possible inviting an expert on auditory hallucinations to a staff in-service and/or having staff visit an ongoing group that deals with managing voices.

\section{Patient Assessment}

Staff on inpatient psychiatric units need to know about each patient's experience of distressing auditory hallucinations with particular attention to whether the voices command the patient to harm themselves or others and whether the patient intends to act on those commands. This information can be obtained by asking patients to complete self-assessment tools about their voices on admission, daily throughout their hospital stay, and upon discharge. Information can also be obtained by interviewing patients about their voices. Interviewing facilitates a patient telling his/her story about hearing voices and also gives staff insight into each patient's individual experience. 
Comprehensive Program Auditory Hallucinations

\section{Evidence-based Assessment Tools}

The authors (RB, LT, \& GD) have developed, tested and refined two patient self-assessment tools that provide information about negative characteristics of auditory hallucinations, intensity of unpleasant voices over the past 24 hours, the presence of commands to harm and intent to act on these commands. These authors have also developed an interview guide to help staff learn more about each person's experience of hearing voices. A brief description of each assessment tool follows with examples of how they can be used on inpatient psychiatric units.

Unpleasant Voices Scale-Inpatient Version (UVS-IP). The Unpleasant Voices Scale (UVS) was designed to help outpatients assess the intensity of their unpleasant hallucinations. This 5item scale asks patients to rate the intensity of their unpleasant voices on a scale of 0 "no voices heard" to 10 "the most unpleasant your voices could be" during the past 24 hours and during the past week, whether they are hearing pleasant voices and command hallucinations to harm self and/or others and whether they intend to act on those commands. The Command to Harm Safety Protocol is enacted if patients respond "yes" to hearing voices commanding them to harm self and/or others and "yes" to intent to act on those voices (Gerlock, Buccheri, Buffum, Trystad \& Dowling, 2009). This outpatient scale was adapted for use on inpatient units by deleting the question that asks patients to rate their voices over the past week and named the UVS-IP.

Command to Harm Safety Protocol. This safety protocol is a tool to guide the course facilitators in assessing patients who have responded "yes" to hearing voices commanding them to harm self and/or others and "yes" to intent to act on those voices on the UVS. The Command to Harm Safety Protocol assesses three major risk areas: 1) expressed intent to hurt self or others; 
Comprehensive Program Auditory Hallucinations

2) the means and the plan to do so and 3) a prior history of hurting self or others (Gerlock, et al., 2009).

Characteristics of Auditory Hallucinations Questionnaire (CAHQ). The Characteristics of Auditory Hallucinations Questionnaire (CAHQ) is a 7-item Likert-type instrument on which patients rate the negative characteristics of their auditory hallucinations (i.e., frequency, loudness, self-control, clarity, tone, distractibility and distress) over the past 24 hours on a scale from 1-5. Higher scores indicate a higher degree of negative characteristics (e.g., more frequent, louder, less self-control) (Buccheri, et al., 2004; Trygstad et al., 2002).

Auditory Hallucinations Interview Guide Inpatient Version (AHIG-IP). The Auditory Hallucinations Interview Guide (AHIG) is a 50-item interview guide developed from the literature and clinical experience that asks patients for demographic and detailed information about auditory hallucinations and commands hallucinations to harm, strategies they have found useful to manage auditory hallucinations and command hallucinations to harm, and psychiatric medication regime (Buccheri et al., 1996). The information elicited from this interview guide about what a patient already does to manage their voices is especially important as patients often say "ask me what I do that works before you try to teach me something else". Clinical utility has been established with the revised and shortened in-patient version (AHIG-IP).

\section{0-Session Course}

During an inpatient psychiatric hospital stay, a patient's effective strategies for managing his/her auditory hallucinations should be reinforced and the patient should be encouraged to learn new strategies as well. Strategies can be taught in a group format or individually if attending a group is not feasible. Learning with others in a group setting is preferable because it 
Comprehensive Program Auditory Hallucinations

allows patients to see that they are not alone and that sharing their experiences can help others.

Other benefits of a group setting include listening to other patients who have found and use

effective strategies to manage their voices and learning to support one another in managing this

symptom (Trygstad, et al, 2002; Yalom, 2005).

\section{Evidence-based Nursing Intervention}

The 10-session Behavioral Management of Persistent Auditory Hallucinations Course was developed based on the UCSF Symptom Management Model (UCSF, 1995), self-monitoring theory (Brier \& Strauss, 1983), and an extensive literature review of strategies to manage auditory hallucinations. The following strategies were taught in the course: self-monitoring, talking with someone, listening to music with or without earphones, watching television, saying "stop"/ignoring what the voices say to do, using an ear plug, relaxation techniques, keeping busy with an enjoyable activity and/or helping others, and practicing communication related to taking medication and not using drugs or alcohol. The 10-session course was tested and found to reduce negative characteristics of auditory hallucinations, anxiety and depression (Buccheri, Trygstad, Kanas, Waldron, \& Dowling, 1996; Buccheri, Trygstad, Kanas, \& Dowling, 1997; Buccheri et al., 2004; Trygstad et al., 2002) as well as intensity of auditory hallucinations and commands to harm (Buccheri, et al, 2007). This course has most recently been successfully taught and found to be effective in six Veterans Health Administration (VA) sites across the US (Buffum, et al., in press), in Canada, Thailand, and Australia. It is currently being taught in many sites in the U.S. and in several other countries including India and China.

Beliefs underlying our approach to stress the value of each person's experience. We believe the environment and context in which we teach the course are as important as the strategies and 
Comprehensive Program Auditory Hallucinations

practice of the strategies. We monitor patient experience and teach specific strategies within a structured atmosphere of respect and support. The achievement of this essential atmosphere is based on the beliefs and behaviors described below.

1. Provide an atmosphere where it is acceptable for patients to admit they hear voices and to talk about what makes them better and worse.

2. Encourage each patient to share his/her experience of hearing voices in the class (many want to talk about the situation when they first heard voices).

3. Let patients know there will be individual variation in their responses to the strategies.

4. Demonstrate each of the strategies in the class and talk about the reasons why they may work.

5. Encourage each patient to practice the strategies in the class before asking them to try them outside the class.

6. Encourage each patient to share his/her responses to each strategy with the class.

Information given to patients at the beginning of the course. We believe that it is important to share what we have learned about teaching self-management of auditory hallucinations with patients at the beginning of the course. Some of the scripted information given to patients during the first class is described below.

1) You are the expert about your voices.

2) We can all learn from one another.

3) You are all individuals--what works for one person may not work for another person.

4) If any strategy makes you uncomfortable, please stop using it or change it to suit your needs.

5) If you have pleasant voices you want to keep, it is not our intention to make them go away. We would like to help you manage the voices that are bothering you. 
Comprehensive Program Auditory Hallucinations

\section{Communicating with Staff}

In order to communicate assessment data about unpleasant voices and commands to harm

with staff, each patient's daily scores from the UVS (e.g., intensity of unpleasant auditory hallucinations, commands to harm and intent to act on commands to harm) can be graphed on the Auditory Hallucinations Checklist (see Figure 1). This checklist can then be placed in the medical record for all staff involved with the care of the patient to read. In order to communicate what occurs in the behavioral management of auditory hallucinations course or groups adapted from the course, staff can complete a template that standardizes the information charted after each class (see Figure 2). Pertinent information from both the checklist and the charting template should be included in shift report so oncoming staff are informed about the intensity of a patient's unpleasant voices, whether he/she hears commands to harm and has intent to act on those commands.

\section{Discharge Communication}

An individualized self-management of auditory hallucinations packet should be included as part of the medical records that accompany a patient upon discharge. This packet should include an assessment tool- the UVS, the safety protocol and a list of the specific strategies that work best for the patient. A brief explanation of the UVS and the safety protocol should also be provided for case managers and/or caregivers. For example, it could be suggested that a patient complete the UVS regularly (e.g., daily or weekly as needed), and be asked to notify his/her case manager and/or caregiver if they are hearing commands to harm and intend to act on those commands. The patient's intent to act on commands to harm would then trigger the initiation of the safety protocol by case managers and/or caregivers. 
Comprehensive Program Auditory Hallucinations

\section{Three Vignettes about Adapting the Program on Different}

\section{Types of Inpatient Psychiatric Units}

Many things dictate how the course is adapted to a specific unit. These factors can include: acuity of patients, length of stay, and staff availability. The following are vignettes that describe three advanced practice nurses' (APNs) experiences in adapting and implementing the program on different types of inpatient units (i.e., locked intensive inpatient psychiatric care unit, acute inpatient psychiatric unit, and mental health rehabilitation center [M.H.R.C.]). Each vignette describes how the nurses adapted the course to their settings and what the nurses did to encourage staff participation.

Vignette one: VA locked 14-bed intensive inpatient psychiatric care unit (KL)

The concept of teaching patients to manage their auditory hallucinations in a group, which began as part of a research project (Buffum, et al., in press), was initiated and continues today on a locked 14-bed intensive inpatient psychiatric care unit at a VA hospital.

\section{Selection of Patients and Staff}

Veterans presenting with a complaint of auditory hallucinations or who are observed by staff responding to hallucinations on the intensive care unit are invited to participate in a twice-weekly group. This group has evolved, in its second year, to also include veterans who present with "intrusive thoughts" regardless of the etiology. This allows more veterans to participate in the group, learning management strategies to deal with their voices/intrusive thoughts. Staff report that broadening the inclusion criteria to include patients who hear intrusive thoughts appears to reduce the stigma associated with attending a managing voices group. 
Comprehensive Program Auditory Hallucinations

At the inception of the group, there were ten psychiatric nurses and one psychiatric occupational therapist that expressed interest and volunteered to participate. The nurses and the occupational therapist came from different educational backgrounds but all expressed an interest in learning how to better serve veterans dealing with auditory hallucinations.

\section{Adaptation of Course to Site}

As an introduction, and in order to increase basic understanding of what a person with auditory or visual hallucinations might be experiencing, the Janssen representative was invited to bring his virtual reality machine to offer the nurses, residents, and attending doctors a chance to experience simulated auditory/visual hallucinations. This had a very profound effect on many of the staff. Some of the comments after participating in the simulated program included:

- "I can't believe they get anything done with all that going on."

- "I feel so overwhelmed with the stimulus."

- "I feel really sad."

- "Unbelievable."

The experience of implementing this group was highly motivating to many of the staff. The inpatient psychiatric staff provided ideas about what appeared to be most useful to the veterans and what needed change in the group format to make it a better fit for the staff, unit schedule, and the veterans participating in the group. The ability of staff to participate in making changes about the format of the group was instrumental in securing the group as a permanent part of the inpatient unit.

During the process of implementing the group, we encountered the following barriers: 1) more time should have been set aside for staff education before the group began; 2) patient 
Comprehensive Program Auditory Hallucinations

resistance and/or anxieties associated with attending the group due the nature of the group (many were too paranoid or were told by their voices not to attend); 3) the unit went through a remodel with resulting physical space limitations; 4) working this new group into the already existing patient education program. In addition, the staff had many concerns surrounding the details of facilitating this new group on an inpatient unit. Many staff expressed concern that the group might make things worse for some of the more fragile veterans. There was also limited availability of experienced facilitators due to changing work schedules. Some of the specific concerns voiced by staff that facilitated the group during the implementation stage included:

- "I don't know how to run this group"

- "I couldn't stop the veteran from getting into his delusion"

- It was difficult to ask one veteran to rate her discomfort with the voices as she stated she felt like we were interrogating her."

The staff also verbalized that they felt the group should be 1 hour not just 50 minutes as it was difficult to get everything done (i.e., review of group rules and homework, discussion and practice of the new strategies as well as time to process any issues, thoughts or feelings that were brought up during the group). It also became apparent that many of the veterans needed additional assistance with the homework. The facilitators found it necessary to use shift-to-shift hand off communication to advise and encourage the evening nurses to help the veterans do their homework. This became such an issue that the homework was revised so that check marks replaced the write in answers. The final homework contained a list of the strategies with check boxes indicating whether the homework was helpful, neutral or bothersome. To encourage daily assessment of the effectiveness of the strategies, a response page was placed in the booklet for 
Comprehensive Program Auditory Hallucinations

each day even those between classes.

We learned that rotating facilitators interrupted the flow of the groups and made continuity difficult. The consensus, after much discussion, was that it was better to have one to two set facilitators with rotating co-facilitators who would also have a chance to eventually learn how to facilitate the group. Initially it was assumed that weekends would be a good time for the group because the weekends were less structured. However, fewer staff members are on duty on the weekends so that restricted available days to weekdays. Due to other competing demands in the inpatient schedule, Tuesday and Thursday were found to be the best days to offer the group.

Because of the need to consolidate, the 10 behavioral strategies were separated into two groups with the self-monitoring skill presented at the beginning of each group. The consolidation of the strategies appeared to work well for the veterans as we found that the veterans liked talking about several strategies each group rather then just one. They seemed to like the idea of being able to decide what strategy worked best for them and that they could try out several different strategies between group days. The final consolidation grouping included: 1) Tuesday techniques--self-monitoring, watching TV, listening to music/radio with or without earphones, earplugs, and relaxation techniques and 2) Thursday techniques included a repeat of self-monitoring, talking with someone, using prescribed medications not drugs or alcohol, saying "stop"/ignoring or not doing what the voices/intrusive thoughts tell you to do, keeping busy doing something I like to do or helping others.

When the criteria for group participation were changed to include veterans with intrusive thoughts, the name of the group was changed to "Persistent Symptoms Management." This name was thought to be less stigmatizing and more representative of all of the veterans now 
Comprehensive Program Auditory Hallucinations

attending the group.

The benefits that were noted upon implementation of the group on the inpatient unit included:

1) The veterans seemed more open to talking about the voices or intrusive thoughts after participating in the group; 2) The nurses verbalized gaining increased awareness about how hearing voices affects the veteran's entire life rather than just solely focusing on whether the voices were commanding in nature and/or increasing the risk of the veteran being a danger to themselves or others.

In addition, based on feedback from the participating staff and veterans who attended the groups, changes were made in both the facilitator and patient manual, including:

1) Combining the strategies to reduce group frequency from 10 days in a row to 4 groups per week then finally 2 groups per week.

2) Introducing the concept of "self-monitoring" at each group--that the veterans preferred to call a "brief check in", which to them meant briefly paying attention to what the voices were saying. The feedback we received was that it was easier to practice this strategy briefly as most patients did not want to focus on the voices/intrusive thoughts too long. The explanation was that for so long the voices had been avoided and the veterans indicated that they did not feel comfortable focusing on the voices or intrusive thoughts for too long.

3) Finally the daily check-in was shortened to a checklist versus a fill in the answer format based on feedback from the staff and veterans.

The significance of incorporating the concept of teaching strategies for managing auditory hallucinations/intrusive thoughts into our inpatient unit turned out to be so much more then just adding a new group. The staff gained a new perspective in working with veterans with persistent 
Comprehensive Program Auditory Hallucinations

auditory hallucinations/intrusive thoughts and in so doing developed a sense of accomplishment in being able to teach those self-management skills. There was also a true exchange of information, cooperation, and coordination as well as a sense of working together to make this a successful group used as part of the treatment of seriously ill veterans. Just as important was the inclusion of veterans in the decision-making about the group. Veterans' input into decisionmaking about the group gave a sense of ownership of the group and seemed to increase their interest and participation.

\section{Vignette two: VA inpatient locked 12-bed psychiatric unit. (MB)}

This experience involved adapting the course to an inpatient locked 12-bed psychiatric unit at Deleted: My Deleted: was in a VA hospital. The patients on this unit had short hospital stays (e.g., 3 days to 12 weeks) so we wanted to provide a rapid approach to teaching the strategies, offer structure to their schedules, encourage some social interaction and feelings of altruism, and engage staff in learning to communicate about auditory hallucinations.

\section{Selection of Patients and Staff}

One of the facilitators, a day shift Psychiatric Clinical Nurse Specialist (CNS), partnered with an evening shift CNS and together worked with all of the staff to adapt the 10-session course with a subset of voice-hearing patients on the unit. Patients were screened using the following criteria: currently experiencing auditory hallucinations, able to sit still in group settings, tolerant Deleted: As Deleted: I

Deleted: we Deleted: We screened Deleted: $p$

Deleted: being of being with others in activities, and interest in attending a group about managing voices. While most voice-hearers were patients with schizophrenia, some had schizoaffective disorder, mania, 
Comprehensive Program Auditory Hallucinations

post-traumatic stress disorder, and/or substance abuse disorders. The facilitators, conducted inservices for all of the staff to learn about the course, strategies, interview guide, communication about hallucinations, and plan for acquisition and location of materials to support the strategies.

\section{Adaptation of Course to Site}

The facilitators taught staff on each shift about all of the strategies, structure for the group, patient assessment tools (CAHQ, UVS), interview guide (AHIG) and resources available on the unit to offer patients (e.g., music as CDs, tapes or radio; ear plugs; reading materials; staff checkout procedures were required for checking out a CD player with ear pieces [related to safety]).

The facilitators demonstrated the group by both of them co-leading with another staff member. The groups were, conducted for 30 to 45 minutes in groups of 3-6 patients four times weekly--twice on day shift and twice on evening shift, alternating days---for close to 1 year.

Patients could come to as many groups as they liked. Patients were encouraged to stay for the entire group but they were allowed them to come and go freely if they were uncomfortable (i.e., too psychotic, paranoid, restless or agitated). The facilitators did not offer the groups sequentially, reviewing different or the same strategies in each group, depending on the needs of group members. In each group, the facilitators taught strategies, practiced strategies, and discussed available resources. Sometimes the groups had to be shorter (only 30 minutes) so that more patients could tolerate the content and contact with others. The small unit size was limiting, as there were often not enough patients who met the criteria for a group. The short stay required creative adaptations -some patients were acutely ill, some less so; they could not always be together for a group because of the range of acuity and severity of illness. Sometimes staff worked individually with patients, and this was often the best strategy for the patient and the
Deleted: We

Deleted: We taught

\begin{tabular}{|l|} 
Deleted: We also \\
\hline Deleted: us \\
\hline Deleted: We
\end{tabular}

Deleted: We encouraged

Deleted: $p$

Deleted: freely

Deleted: We
Deleted: many

Deleted: with schizophrenia

\begin{tabular}{|l|} 
Deleted: We \\
Deleted: s \\
Deleted: , related to
\end{tabular}


Comprehensive Program Auditory Hallucinations

acuity of the inpatient setting.

We used two patient assessments tools (CAHQ, UVS) and the interview guide (AHIG), homework and lists of strategies in the groups. Related to illness, fatigue, medication, or a combination of these, patients were sometimes unable to think clearly. We realized in helping patients complete assessment tools and homework, that some of them had difficulty seeing, were unable to read, or had difficulty with English. Also, some patients had difficulty with the UVS because the numeric rating did not make conceptual sense to them. Most patients had never rated their voices before. This took some staff explanation and practice to conceptualize how to rate this particular symptom on a scale of $0-10$.

The AHIG was invaluable in facilitating a relationship between the admitting nurse and the patient. The interview provided a forum for each patient to talk about his/her voices and the nurse learned about the patient's unique experience. For example, staff learned that many patients like their pleasant voices as company, so they do not want to lose them but would very much like to have their unpleasant voices go away. Sometimes the completion of the AHIG had to be done over several sessions because patients could not tolerate a long interview. Another benefit of using the AHIG was that the nurse could share what they learned with other staff caring for that patient. Staff could then look for specific signs of distress, offer what was already identified as being helpful, and generally be more attentive to a patient's needs. This included offering the patient a list of strategies and other resources available on the unit.

As a result of attending the groups, patients did not have to feel so alone with their symptom. They learned to talk with staff and with other patients in their groups about their voices. Patients could share strategies, and help each other---altruism is easily activated in the VA. Having 
Comprehensive Program Auditory Hallucinations

served in the military, many patients are accustomed to "watching out for their fellow soldiers."

Additionally, some veterans described in the group that they had never talked about their voices before, that they felt staff seemed genuinely interested and wanted to learn about their experiences, and that they liked being able to talk with other patients with whom they knew they had this commonality. Indeed, patients were observed sharing strategies with one another both within and outside the group.

Vignette three: : Private 88-bed locked M.H.R.C. (RB)

This course has been taughtat a M.H.R.C. twice a year for the past 6 years. This is a private 89bed locked facility for psychiatric patients, many of whom have a dual diagnosis of chronic mental illness and substance abuse. The patients are usually referred to the M.H.R.C. from acute psychiatric units since they are not stable enough to be discharged to the community.

\section{Selection of Patients and Staff}

Names of patients who might benefit from the course are solicited from staff. It is often surprising which patients actually attend do the homework and turn it in each week. There are usually 1-2 patients who are a surprise to the staff that they not only attended but completed and returned their homework.

The same person has been the primary facilitator of the course over the six-year period but the

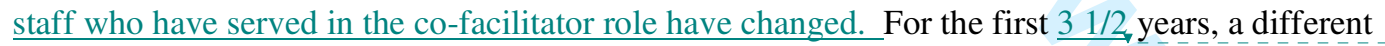
staff member co-facilitated the course each time the course was taught. The idea was to familiarize as many staff as possible with the course. For the past $11 / 2$ years, the same staff member, who is especially dedicated to helping people with their voices, has co-facilitated the course The benefits of having a consistent co-facilitator are that that staff member knows the
Deleted: Setting three

Deleted: My experience has been in

Deleted: teaching the course

\begin{tabular}{l} 
Deleted: I solicit from staff the \\
\hline Deleted: $\mathrm{n}$ \\
\hline Deleted: o \\
\hline Deleted: s \\
Deleted: and actually \\
Deleted: es \\
Deleted: s \\
Deleted: eople \\
Deled: and myself \\
\hline
\end{tabular}

Deleted: person

Deleted: with me

Deleted:

Deleted: past 3

Deleted: times

Deleted: person

Deleted: with me

Deleted: she 
Comprehensive Program Auditory Hallucinations

patients and starts to encourage those who have distressing voices to attend long before they are formally invited to attend the course. Additonally, extra homework forms can be left with the co-facilitator in case patients, lose their form In addition, the co-facilitator is available to help the patients during the week with their homework.

\section{Adaptation of Course to Site}

This course was adapted to this setting by shortening each class from 50 minutes to 30 minutes (patients have busy schedules and their attention span is less than that of outpatients), teaching one class per week for six week due to the facilitator's academic schedule, and including the strategies where supplies were unnecessary (1. self-awareness or what makes the voices better and worse [formerly called self-monitoring but was changed as many patients did not understand the term]; 2. talking with someone; 3. relaxation; 4. watching TV or something that moves; 5. keeping busy doing something I like to do or helping others; 6. taking prescribed mediations and saying "no" to drugs and alcohol). Since it is a short class, only some of the patients can share the results of their UVS and read the directions on the homework. There is competition among patients for this activity. Many of them enjoy sharing their experiences and reading the homework forms aloud. Patients who graduate are often encouraged to attend a second six-week course to reinforce their use of the strategies and several have been invited tobe a peer facilitator to recognize their expertise. All patients receive healthy snacks at the end of each class (patients who return their homework receive a reward such as a $\$ 1$ or a cookie Deleted: Often I have encouraged Deleted: $p$ Deleted: have let two of them Deleted: Deleted: my assistant Deleted: an additional Deleted: snack depending on what is available). Signs are posted on the unit announcing "the strategy of the week" and a copy of the course treatment manual is left at the nursing station and the UVS and 
Comprehensive Program Auditory Hallucinations

homework forms are posted on the staff bulletin board. A certificate of completion is given during the last class if patients have attended 4 of 6 classes.

The facilitator has done two staff in-services on managing distressing auditory hallucinations at this facility-the first to introduce the topic to staff and the second one with two staff who cofacilitated the group Names of patients who have attended are announced in report and at the end of the course, the names of patients who are graduating and receiving a certificate are also announced. All levels of staff seem to enjoy hearing about who is graduating. Discussion

This paper describes a comprehensive program for assessing and managing auditory hallucinations that can be adapted for use on inpatient psychiatric units. The core of this comprehensive program is evidence-based; the adaptations to specific sites utilized the expert clinical judgment of advanced practice nurses and required flexibility on the part of all staff. Implementing research into practice at real sites requires adaptation to site, patients, staff and unit realities (e.g., short stays, small group sizes). Tips to help in the adoption of this program are summarized in Table 1.

Patients need to be able to adapt the program for themselves. Self-management ultimately means adaptation of the program to self. To incorporate this point of view in the course, patients are always told in the first class that they are the experts on what works for them. If a selfmanagement strategy makes them uncomfortable, they should not do it or should modify the strategy to suit them. Within the course, patients will have varied responses to the selfmanagement strategies. We encourage the sharing of different experiences. We teach selfmanagement strategies and it is our experience that everyone who completes the course finds one

Deleted: and
Deleted: a
Deleted: they
Deleted: I have
Deleted:
Deleted: one by myself
Deleted:
Deleted:
Deleted: a
Deleted: with me
Deleted: I announce in report the
Deleted: $n$
Deleted: I also post signs on the unit
announcing "the strategy of the week"
and have put a copy of the course
treatment manual at the nursing station
and posted the UVS and homework forms
on the staff bulletin board. $I$

Deleted: we

Deleted: ell patients 
Comprehensive Program Auditory Hallucinations

or more strategies that work for them. Every strategy is adopted by at least one patient in the course and no one strategy works for everyone in the course.

For patients with schizophrenia, distressing auditory hallucinations including commands to harm and intent to act on those commands should be the sixth vital sign. Shift report should include the intensity of a patient's unpleasant voices on a scale of 0-10 during the previous shift, whether the patient hears commands to harm and if they plan to act on them (i.e., information all available from UVS). Just as we include elevated vital signs in end of shift report, information about auditory hallucinations, especially commands to harm should be part of the inter and intra shift communication. This is consistent with a sixth vital sign that has been proposed specific to several nursing specialties (e.g., emotional distress for oncology patients, dyspnea for COPD patients [Bultz, \& Carlson, 2005; RNAO, 2005]).

\section{Implications for Practice}

To improve patient care and safety, nurses on inpatient psychiatric units are encouraged to adapt and implement this comprehensive program to assess and manage distressing auditory hallucinations and commands to harm. This program uses assessment data to monitor and individualize patient care by teaching patients to practice a variety of strategies and then asking them to select and use those strategies that are most effective in helping them self-manage their auditory hallucinations. Above all, the program enables optimal communication about voices between staff and patients. In all adaptations of the course presented in this paper, staff became more comfortable initiating discussions with patients about voices. This program also encourages the sharing of assessment tools and effective self-management strategies with case managers and/or caregivers when patients are discharged. 
Comprehensive Program Auditory Hallucinations

1

2

3

4

5

6

7

8

9

Outcome tools and data collection are needed to evaluate and improve psychiatric inpatient care. It is not known what the outcomes of inpatient psychiatric hospitalization are on auditory hallucinations and commands to harm. How much do the average patient's unpleasant auditory hallucinations decrease in intensity after an inpatient psychiatric hospital hospitalization? Do most patients experience a decrease in the negative characteristics of their auditory hallucinations after hospitalization? If so, how much of a decrease and how long does it last? How many patients no longer hear commands to harm after an inpatient psychiatric hospitalization? This kind of outcome data will be very important in evaluating and improving current inpatient practice in managing auditory hallucinations.

Specifically the overall effectiveness of inpatient treatment of distressing auditory hallucinations and commands to harm, could be measured using the assessment tools (i.e., UVS and CAHQ) that are part of this comprehensive program. A comparison of the admission and discharge scores from the UVS could measure improvement in intensity of unpleasant auditory hallucinations and commands to harm. A comparison of admission and discharge scores from the CAHQ could measure improvement in seven negative characteristics (i.e., frequency, loudness, self-control, clarity, tone, distractibility and distress) of auditory hallucinations.

\section{Implications for Future Research}

The core of this comprehensive program has been tested and found to be effective. The next phase of our research will be to test this program of assessing and managing auditory hallucinations from admission to discharge on inpatient psychiatric units by measuring patient outcome data using the UVS and CAHQ as well as staff ratings of helpfulness for each component of the program. 
Comprehensive Program Auditory Hallucinations

Another implication for future research is to incorporate the role of a peer counselor into the course and to evaluate perceived benefits and helpfulness of the peer counselor by patients and staff. The importance of this has been highlighted by the VA that has focused part of their mental health research agenda on the benefits of peer counseling: "Assess the effectiveness of peer-support programs in supporting recovery and community reintegration in veterans with mental illness" (HSR\&D, 2008).

_ Research has demonstrated that a peer counselor can be effective in working with people with schizophrenia on inpatient psychiatric units (Rummel-Kuge, Stiegler-Kotzor, Schwarz, Hansen, \& Kissling, 2008). These researchers evaluated using a peer counselor with 88 inpatients with schizophrenia. The peer counselor was available for one hour a week for 20-30 minute individual consultation sessions with psychiatric inpatients with a diagnosis of schizophrenia. Patients reported that the peer counselor was able to answer their questions, that they felt like they were understood by the peer counselor, and that he provided them with support and encouragement. Ninety-six percent of the patients would recommend peer counseling to others. The researchers concluded that peer counseling is feasible and effective with inpatients with schizophrenia. 
Comprehensive Program Auditory Hallucinations

1

2

3

4

5

6

7

8

9

10

11

12

13

14

15

16

17

18

19

20

21

22

23

24

25

26

27

28

29

30

31

32

33

34

35

36

37

38

39

40

41

42

43

44

45

46

47

48

49

50

51

52

53

54

55

56

57

58

59

60

\section{References}

Author. (March, 2005). Best practice guideline.-Shaping the future for nursing. Nursing care of dyspnea: The 6th vital sign in individuals with chronic obstructive pulmonary disease nursing. Ontario, Toronto: Registered Nurses Association of Ontario (RNAO).

Buccheri, R., Trygstad, L., \& Dowling, G. (2007). Behavioral management of command hallucinations to harm in schizophrenia. Journal of Psychosocial Nursing and Mental Health Services, 45(9), 46-54.

Buccheri, R., Trygstad, L., Dowling, G., Hopkins, R., White, K., Griffin, J., et al. (2004). Long term effects of teaching behavioral strategies for managing persistent auditory hallucinations in schizophrenia. Journal of Psychosocial Nursing, 42(1), 18-27.

Buccheri, R. K., Trygstad, L., Kanas, N., \& Dowling, G. (1997). Symptom management of auditory hallucinations in schizophrenia: Results of 1-year follow up. Journal of Psychosocial Nursing and Mental Health Services, 35(12), 20-28.

Buccheri, R. K., Trygstad, L., Kanas, N., Waldron, B., Dowling G. (1996). Auditory hallucinations in schizophrenia: Group experience in examining symptom management and behavioral strategies. Journal of Psychosocial Nursing and Mental Health Services, 34, 1225. 
Comprehensive Program Auditory Hallucinations

Buffum, M., Buccheri, R., Trygstad, L., Gerlock, A., Dowling, G., Birmingham, P., \& Kuhlman, G. (in press). Implementing evidence-based practice: Educating nurses to conduct the behavioral management of auditory hallucinations course. Journal of Psychosocial Nursing and Mental Health Services.

Bultz, B. D., \& Carlson, L. E. (2005). Emotional distress: The sixth vital sign in cancer care. Journal of Clinical Oncology, 23(26), 6440-644.

Deggan, P. (n.d.). Hearing voices curriculum: Complete training and curriculum package. Lawrence, MA: National Empowerment Center.

Gerlock, A., Buccheri, R., Buffum, M., Trygstad, L., \& Dowling, G. (2009). Tools to respond to command hallucinations to harm self and others: Unpleasant Voices Scale and Safety Protocol. Manuscript submitted for publication.

HSR\&D [Veterans Affairs]. (2008). Program announcement: HSR\&D priorities for investigator -initiated research. Retrieved June 15, 2009, from http://www.research.va.gov/funding/solicitations/docs/HSRD-IIR-Priorities-2008-09.pdf

Rummel-Kluge, C., Stiegler-Kotzor, M., Schwarz, C., Hansen, W.-P., \& Kissling, W. (2008). Peer-counseling in schizophrenia: Patients consult patients. Patient Education and Counseling, 70, 357-362.

Trygstad L., Buccheri, R., Dowling, G., Zind, R., White, K., Griffin, J., et al. (2002). Behavioral management of persistent auditory hallucinations in schizophrenia: Outcomes from a 10-week course. Journal of the American Psychiatric Nurses Association, 8(3), 8491.

U.C.S.F. Symptom Management Faculty Group. (1995). A model for symptom management. 


\section{Page 25 of 28}

Comprehensive Program Auditory Hallucinations

1

2

3

4

5

6

7

8

9

10

11

12

13

14

15

16

17

18

19

20

21

22

23

24

25

26

27

28

29

30

31

32

33

34

35

36

37

38

39

40

41

42

43

44

45

46

47

48

49

50

51

52

53

54

55

56

57

58

59

60

The Journal of Nursing Scholarship, 26, 272-276.

Yalom, I.D. (2005). The theory and practice of group psychotherapy (5th ed.). New York:

Basic Books.

URL: http://mc.manuscriptcentral.com/umhn Email: sthomas@utk.edu 
Comprehensive Program Auditory Hallucinations

Figure 1

Charting Checklist for Auditory Hallucinations

(14 days)

Directions: 1. Put the date in the boxes across the top.

2. Put an dot in the middle of the box for scores on the Unpleasant Voices Scale, question \#1 then connect the dots to graph the scores.

3. Put "Y" for yes and " $\mathrm{N}$ " for no in the rest of the boxes.

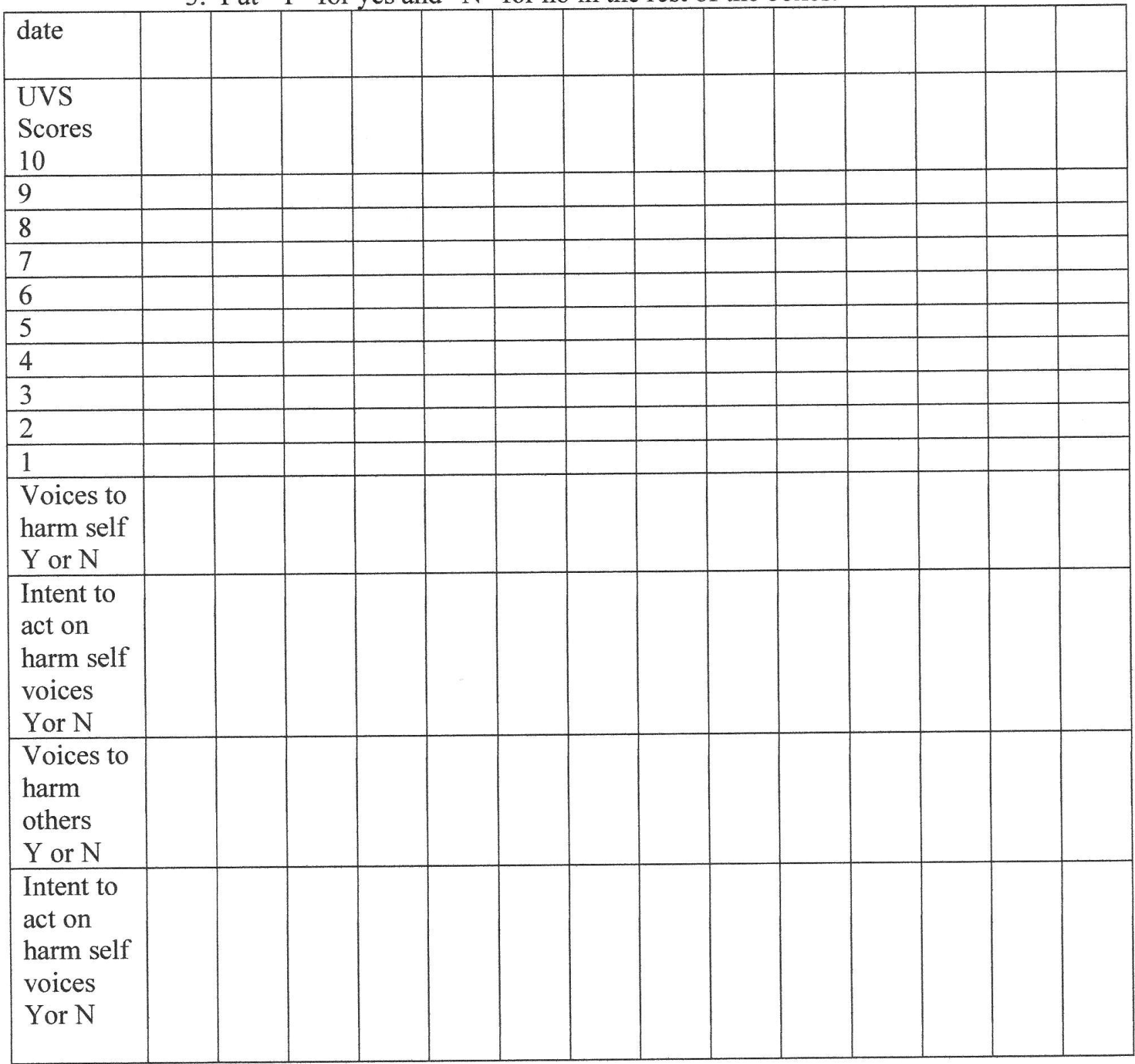


Figure 2

Charting Template

The following is a suggested format for charting using a Subjective/Objective, Assessment, and Plan (SOAP) model:

Behavioral Management of Anxiety and Auditory Hallucinations -- Class \#

This participant was one of participants who attended Class\# _ Behavioral Management of Anxiety and Auditory Hallucinations Course. This class was facilitated by and

$\underline{\mathrm{S} / \mathrm{O}}$ : Class Focus: our focus today was

\section{Voices assessment:}

1) Participants completed the Unpleasant Voices Scale (UVS/1-10)

Unpleasant voices over the past 24 hours:

Answered yes or no to command hallucinations to harm self.

Answered yes or no to intent to harm self.

Answered yes or no to command hallucinations to harm others.

Answered yes or no to intent to harm others.

The safety protocol was or was not instituted

New strategy for the week was introduced: “ " and why it may be helpful. The participants practiced the technique and discussed.

$\underline{A / P}$ : Describe behavior in group. Discuss if the patient had command hallucinations to harm self or others and intent to act on them and if you had to institute the safety protocol or anything else that should be noted. They were given take-home work for the next week. The class will next meet 
Comprehensive Program Auditory Hallucinations

Table 1

Tips for Successfully Adopting Comprehensive Program for Self-management of Auditory Hallucinations

1) Provide a reading list of articles with patients' accounts of their experience of hearing voices and/or do a simulated voices exercise with staff to increase awareness and begin discussion of current assessment and treatment of auditory hallucinations.

2) Share articles, course treatment manual, instruments and homework forms with all staff on unit.

3) Have as many staff as possible be involved in the decision to implement new program.

4) Include entire inpatient staff in training sessions so they can reinforce what patients are learning.

5) Conduct ongoing staff evaluation sessions to determine adaptations, consistency in utilization of course tools, experiences with conducting the course, perspectives about patient benefits, and ideas for sharing information with all unit staff.

6) Have as many staff as possible sit in and observe the classes (e.g, 2 staff per class for 10 classes would be 20 staff members).

7) Use charting template to save time and to communicate in a consistent way with other staff.

8) Customize the discharge plan to include patient responses to self-management strategies.

9) Send tools and safety protocol to caregivers when they are discharged.

10) Develop graduate group to bridge inpatient and outpatient treatment. 